\title{
Cardiopulmonary performance in allogeneic hematopoietic cell transplantation recipients - evaluation of pre-transplant risk assessments
}

\author{
Antonia Pahl $\mathbb{D}^{1} \cdot$ Sarah Waibel $\mathbb{C}^{2} \cdot$ Anja Wehrle ${ }^{3} \cdot{\text { Gabriele Ihorst } \mathbb{D}^{4} \cdot \text { Albert Gollhofer }^{3} \cdot \text { Hartmut Bertz }}^{1}$
}

Received: 28 July 2020 / Revised: 2 December 2020 / Accepted: 4 December 2020 / Published online: 6 January 2021

(c) The Author(s) 2021. This article is published with open access

\begin{abstract}
Cardiopulmonary performance reflects how well different organ systems interact. It is inter alia influenced by body composition, determines patients' quality of life and can also predict mortality. However, it is not yet used for risk prediction prior to allogeneic hematopoietic cell transplantations (alloHCT). Thus, we aimed to examine the predictive power of peak oxygen consumption (VO2peak) as a representative of cardiopulmonary performance and that of body composition before alloHCT to determine overall survival (OS) and non-relapse mortality (NRM) 2 years after transplantation. We also compared it with the predictive power of four commonly-used risk scores: revised Pretransplant Assessment of Mortality (rPAM), Hematopoietic Cell Transplantation-specific Comorbidity Index (HCT-CI), revised Disease Risk Index (rDRI), European Society for Blood and Marrow Transplantation (EBMT). Fifty-nine patients performed a cardiopulmonary exercise test and body composition assessments before alloHCT and were observed for 2 years. Sixteen patients died. VO2peak and most risk scores assessed pre-transplant revealed no association with OS or NRM. Body composition parameters only within univariable analyses. But higher rDRI and the male sex, were associated with shorter OS and higher NRM. We thus propose that the current risk assessments be reconsidered. The predictive value of VO2peak and body composition need further clarification, however.
\end{abstract}

\section{Introduction}

In recent decades, the number of allogeneic hematopoietic cell transplantations (alloHCT) has risen [1] and due to the use of reduced intensity conditioning protocols, alloHCT can be offered to older patients and those with more comorbidities [2,3]. Pre-transplant assessments enable an individual risk-benefit ratio, as all-cause mortality still amounts to $46 \%$ [3]. In this context, calculating individual

Antonia Pahl

antonia.pahl@uniklinik-freiburg.de

1 Department of Medicine I, Faculty of Medicine and Medical Center, University of Freiburg, Freiburg, Germany

2 Department of Neurology and Neuroscience, Faculty of Medicine and Medical Center, University of Freiburg, Freiburg, Germany

3 Department of Sport and Sport Science, University of Freiburg, Freiburg, Germany

4 Clinical Trials Unit, Faculty of Medicine and Medical Center, University of Freiburg, Freiburg, Germany risk scores to predict overall survival (OS) and non-relapse mortality (NRM) are used prior to alloHCT. Commonly used scores are $[4,5]$ the European Blood and Bone Marrow Transplantation (EBMT) score [6], the Hematopoietic Cell Transplantation-Comorbidity-Index (HCT-CI) [7] the revised Disease Risk Index (rDRI) [8] and the revised Pretransplant Assessment of Mortality score (rPAM) [9]. As none of these scores covers all important variables, the concurrent use of various scores is recommended to include various aspects of risk prediction. However, according to an analysis by Shouval et al. [5] the forecast reliability of those scores ranges from moderate to random. To specify the individual risk-benefit ratio and provide an additional basis for medical decisions, e.g., the intensity of conditioning therapy, patients' organ functions are usually determined by medical tests. The body mass index (BMI) is also calculated. Although muscle weakness and restrictions of the cardiorespiratory system are common therapy related side effects [10-13] that may cause mortality [14, 15], so far the level of physical performance is not studied in this context. Stress tests could therefore help us estimate the interaction of different organ's function and allow more accurate 
assessment of patients' physical performance. A cardiopulmonary exercise test (CPET) covers pulmonary, cardiovascular, blood circulation, and muscle systems' interaction, thereby providing a comprehensive evaluation of a patient's physical performance and organ function under stress. Peak oxygen intake (VO2peak) as a main CPET outcome is known to predict mortality in healthy individuals [16] and in breast or colon cancer survivors $[17,18]$. There are initial indications that pre-alloHCT VO2peak could also provide information for predicting OS and NRM $[19,20]$. But these results have not yet been confirmed or compared to existing risk calculation methods. CPET outcomes are also influenced by patients' body composition parameters [21, 22]; the phase angle in particular also predicts clinical outcomes [23, 24]. However, the validity of these individual risk prediction methods-the calculation and use of risk scores, examining physical functioning and body composition parameters-have not been concurrently verified within one sample.

Aim of the present analysis was therefore to examine the explanatory power of pre-alloHCT VO2peak and body composition to predict OS and NRM 2 years after transplantation. We also compared this risk prediction to those of commonly-used risk scores. We hypothesized that assessing VO2peak and body composition would improve the present risk prediction methods and thus enable a more accurate post-alloHCT prognosis.

\section{Materials and methods}

\section{Study design and patients}

This survival analysis relied on the baseline data from a randomized controlled trial [25]. This study was designed to investigate the effects of whole body vibration training on patient's physical performance during alloHCT. Examinations were performed before conditioning therapy, at hospital discharge and 180 days after alloHCT. Within a 17-month period, patients scheduled for alloHCT were consecutively recruited at the Department of Medicine I, University Medical Center Freiburg, Germany on the day of hospital admission. Included patients underwent study assessments prior to their first administration of conditioning therapy including CPET and body composition analysis in the course of baseline procedure. More information about the study design and inclusion and exclusion criteria can be found elsewhere [25]. For OS- and NRM-analysis, patients were followed for 2 years after alloHCT. Only patients with hematological malignancies were included in the survival analysis. 


\section{Cardiopulmonary exercise test}

VO2peak was examined during CPET. Patients performed an incremental CPET on an electronically-braked cycle ergometer (ergoline ergoselect 1200, Ergoline GmbH, Bitz, Germany) in recumbent $\left(40^{\circ}\right)$ position during continuous monitoring of their electrocardiogram, heart rate, and blood pressure. Gas exchange and ventilation were recorded continuously via breath-by-breath gas analysis (MetaLyzer 3BR3, Cortex Biophysik GmbH, Leipzig, Germany). Patients had to perform until exhaustion starting at $20 \mathrm{~W}$ with a $10-\mathrm{W}$ increase every minute. Exhaustion was determined by respiratory exchange ratio (RER) $\geq 1.1$ or a heart rate $\geq 85 \%$ of the age predicted maximum [26, 27]. The RER describes the ratio of carbon dioxide produced and oxygen consumed. It is an indicator of metabolic fuel and therefore rates patients metabolic effort [21]. VO2peak describes the maximum amount of oxygen uptake during CPET, and is influenced by pulmonary, cardiovascular, hematopoietic, neuropsychological and muscular functioning and interaction [21, 28]. We also documented the data on maximum reached watt (Pmax) and received perception exertion (RPE). RPE also reflects patients' effort rated subjectively on a scale from 6 to 20 immediately upon discontinuing CPET [29].

\section{Body composition}

BMI was calculated as body weight $(\mathrm{kg})$ divided by squared height $\left(\mathrm{m}^{2}\right)$. Bioelectrical impedance analysis was used to determine body fat mass (FM) (\%), lean body mass (LBM) $(\%)$, body cell mass $(\mathrm{BCM})(\%)$, and phase angle $\left({ }^{\circ}\right)$ (BIA, Nutriguard-S, Data Input, Pöcking, Germany). This measurement can reveal cell membrane function, and thus evaluates body composition in more detail [30]. We applied sex-, age-, and BMI-specific references [31] to calculate the standardized phase angle according to [23]: $\frac{\text { phase angle-references }}{\text { standard deviation references }}$. To ensure better validity, all measurements were taken in the morning after at least $20 \mathrm{~min}$ rest, in lying position and before breakfast [32].

\section{Risk scores and clinical parameters}

We calculated the EBMT score and HCT-CI prospectively before alloHCT, whereas the rPAM score and the rDRI were calculated retrospectively for this analysis. Information on the contents of the individual scores, their range and the classification of the risk groups can be found in Table 1 . For our analysis we have combined the low and intermediate risk group of the rDRI. Information on OS and NRM was collected during medical follow-up examinations. Medical characteristics and history were retrospectively extracted from medical reports.

\section{Quality of life}

We used the subscales "global quality of life (QoL)" and "physical function" of the EORTC QLQ-C30-questionnaire to supplement description of patients' physical functioning and for survival analysis.

\section{Statistical analyses}

Patients' characteristics and physical conditioning were described using absolute and relative frequencies or median (range) respectively. Since all patient data were observed over exactly 24 months, we evaluated differences between 2-year-survivors $(2 \mathrm{yrS})$ and 2-year-decedents $(2 \mathrm{yrD})$ via nonparametric tests. Relapse was considered as a competing risk. Patients still alive after two years of observation were censored at this time. The Kaplan-Meier method was used to estimate the probability of survival. Cox's method was used for univariable and multivariable analyses on OS. Analyses on NRM were done by Fine \& Gray model. Results are presented as hazard ratios (HR) for Cox's model and sub-distribution HR (SHR) for the Fine \& Gray model, together with two sided $95 \%$ confidence intervals (CI). For continuous prognostic variables, the HR/SHR describes the risk increase for a one unit (or unit specified in the table) increase in the prognostic variable. The variable selection for multivariable models is based on profound theoretical considerations, including univariable results and possible correlations between prognostic factors to reduce multicollinearity. Gender, remission, conditioning, the EBMT score, rDRI and HCT-CI were examined as categorical variables. The Karnofsky Performance Scale (KPS) was categorized in below and above or equal 90\%. All other parameters were calculated as continuous variables. The proportional hazards assumption was tested using graphic methods. Statistical analyses were conducted using the IBM SPSS Version 25 (SPSS Inc., Chicago, Illinois, USA) and SAS 9.4 (SAS Institute Inc., Cary, NC, USA) software.

\section{Results}

We enrolled a sample of 71 patients in this study [25]. Data on 59 patients were used for survival analysis: four patients had to be excluded due to a nonmalignant disease, two died before alloHCT, medical data were missing on one patient, and five patients were unable to perform CPET (four patients due to inappropriate blood values, one due to a respiratory infection). All patients received peripheral blood stem cells from an unrelated donor. Patients' characteristics is presented in Table 2 . 
Table 2 Patient characteristics.

\begin{tabular}{|c|c|c|c|c|}
\hline & All $N=59$ & $2 \mathrm{yrS} N=43$ & $2 \mathrm{yrD} N=16$ & $P$ value \\
\hline Age $[\text { years }]^{\mathrm{a}}$ & $56(19-78)$ & $54(19-75)$ & $61(31-78)$ & 0.063 \\
\hline \multicolumn{5}{|l|}{$\operatorname{Sex}[n]^{\mathrm{b}}$} \\
\hline Male:female & $40: 19(68: 32)$ & $27: 16(63: 37)$ & $13: 3(81: 19)$ & \\
\hline Disease $[n]^{\mathrm{b}}$ & & & & 0.715 \\
\hline Myeloid $^{\mathrm{c}}$ & $46(78)$ & $34(79)$ & $12(75)$ & \\
\hline Lymphatic $^{\mathrm{d}}$ & $15(22)$ & $9(21)$ & $4(25)$ & \\
\hline Remission at alloHCT $[n]^{\mathrm{b}}$ & & & & 0.591 \\
\hline CR/chronic phase & $27(46)$ & $23(54)$ & $4(25)$ & \\
\hline Not in CR & $32(54)$ & $20(46)$ & $12(75)$ & \\
\hline HLA-mismatch $[n]^{\mathrm{b}}$ & & & & 0.650 \\
\hline None $(10 / 10)$ & $36(61)$ & $27(62.8)$ & $9(56.3)$ & \\
\hline Mismatch $(\leq 9 / 10)$ & $23(39)$ & $16(37.2)$ & $7(43.8)$ & \\
\hline Gender donor: recipient & & & & 0.964 \\
\hline Female:male & $15(25)$ & $11(26)$ & $4(25)$ & \\
\hline All other & $44(75)$ & $32(74)$ & $12(75)$ & \\
\hline Conditioning $[n]^{\mathrm{b}}$ & & & & 0.168 \\
\hline Myeloablative & $15(25.4)$ & $12(27.9)$ & $3(18.8)$ & \\
\hline$T T / B U / F L U$ & $12(20.3)$ & $9(21)$ & $3(18.8)$ & \\
\hline$T B I / V P 16$ & $1(1.7)$ & $1(2.3)$ & 0 & \\
\hline$T B I / T T$ & $1(1.7)$ & $1(2.3)$ & 0 & \\
\hline$F L U / B U 4$ & $1(1.7)$ & $1(2.3)$ & 0 & \\
\hline Reduced intensity & $44(74.6)$ & $31(72.1)$ & $13(81.2)$ & \\
\hline FLU/BCNU/MEL & $14(23.7)$ & $10(23.3)$ & $4(25)$ & \\
\hline FLU/BCNU/TT & $1(1.7)$ & $1(2.3)$ & 0 & \\
\hline FLU/TT/MEL & $28(47.5)$ & $20(46.5)$ & $8(50)$ & \\
\hline FLU/TT mod. + TREOS & $1(1.7)$ & 0 & $1(6.2)$ & \\
\hline 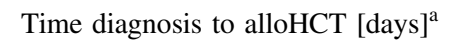 & $214(51-2569)$ & $187(51-2569)$ & $550(85-1702)$ & 0.068 \\
\hline $\mathrm{KPS}[\%]^{\mathrm{a}}$ & $90(70-100)$ & $90(70-100)$ & $90(70-100)$ & 0.375 \\
\hline HCT-CI $[n]^{\mathrm{b}}$ & & & & 0.550 \\
\hline Low risk (0) & $17(29)$ & $13(30)$ & $4(25)$ & \\
\hline Intermediate risk (1-2) & $20(34)$ & $15(35)$ & $5(31)$ & \\
\hline High risk $(\geq 3)$ & $22(37)$ & $15(35)$ & $7(44)$ & \\
\hline EBMT score $[n]^{\mathrm{b}}$ & & & & 0.768 \\
\hline Low risk $(0-2)$ & $7(12)$ & $5(12)$ & $2(13)$ & \\
\hline Intermediate risk (3-5) & $36(61)$ & $27(63)$ & $9(56)$ & \\
\hline High risk (6-7) & $16(27)$ & $11(25)$ & $5(31)$ & \\
\hline $\operatorname{rDRI}[n]^{\mathrm{b}}$ & & & & 0.139 \\
\hline Low risk & $1(1.7)$ & $1(2.3)$ & 0 & \\
\hline Intermediate risk & $32(54.2)$ & $24(55.8)$ & $8(50)$ & \\
\hline High risk & $18(30.5)$ & $16(37.2)$ & $2(12.5)$ & \\
\hline Very high risk & $8(13.6)$ & $2(4.7)$ & $6(37.5)$ & \\
\hline rPAM score [score] ${ }^{\mathrm{a}}$ & $23.9(12.1-42.1)$ & $22.9(12.1-33.5)$ & $25(16.4-42.1)$ & 0.192 \\
\hline Acute GVHD $[n]^{\mathrm{b}}$ & & & & 0.687 \\
\hline None & $29(49.2)$ & $21(48.8)$ & $8(50)$ & \\
\hline Grade I & $11(18.6)$ & 9 (20.9) & $2(12.5)$ & \\
\hline Grade II & $6(10.2)$ & $6(14)$ & 0 & \\
\hline Grade III & $11(18.6)$ & 5 (11.6) & $6(37.5)$ & \\
\hline
\end{tabular}


Table 2 (continued)

\begin{tabular}{llll}
\hline & All $N=59$ & $2 \mathrm{yrS} N=43$ & $2 \mathrm{yrD} N=16$ \\
\hline $\begin{array}{l}\text { Grade IV } \\
\text { Chronic GVHD }[n]^{\mathrm{b}}\end{array}$ & $2(3.4)$ & $2(4.7)$ & 0 \\
$\quad$ None & $32(54.2)$ & $24(55.8)$ & $8(50)$ \\
Limited & $8(13.6)$ & $8(18.6)$ & 0 \\
Extensive & $11(18.6)$ & $11(25.6)$ & 0 \\
Missing data ${ }^{\mathrm{e}}$ & $8(13.6)$ & 0 & $8(50)$ \\
Causes of death $[n]^{\mathrm{b}}$ & & & 0 \\
Alive & $43(72.9)$ & $43(100)$ & $4(25)$ \\
Relapse/progression disease & $4(6.8)$ & 0 & $1(6.3)$ \\
Organ failure & $1(1.7$ & 0 & $5(31.3)$ \\
GVHD & $5(8.5)$ & 0 & $6(37.5)$ \\
Infection & $6(10.2)$ & 0 & 0
\end{tabular}

2yrs $S$ 2-year survivor, 2yrsD 2-year decedents, $H L A$ human leukocyte antigen, $T T$ thiotepa, $B U$ busulfan, $F L U$ fludarabine, $T B I$ total body irradiation, VP16 etoposide, $B C N U$ camustin, $M E L$ melphalan, TREOS treosulfan, $A L L$ acute lymphocytic leukemia, $C R$ complete remission, $K P S$ Karnofsky Performance Scale, HCT-CI Hematopoietic Cell Transplantation-specific Comorbidity Index, EBMT European Society for Blood and Marrow Transplantation, $r D R I$ revised Disease Risk Index, $r P A M$ revised Pretransplant Assessment of Mortality, GVHD graft-versus-host disease.

${ }^{\mathrm{a}}$ Median (range).

bercentage of $N(\%)$.

'Includes: acute myeloid leukemia, chronic myeloid leukemia, chronicle myelomonocytic leukemia, osteomyelofibrosis and myelodysplastic syndrome.

dIncludes: hodgkin disease, non-hodgkin lymphoma, multiple myeloma, chronic lymphatic leukemia, acute lymphatic leukemia.

${ }^{e}$ Patients had died by the time chronic GVHD can occur (100 days after alloHCT).

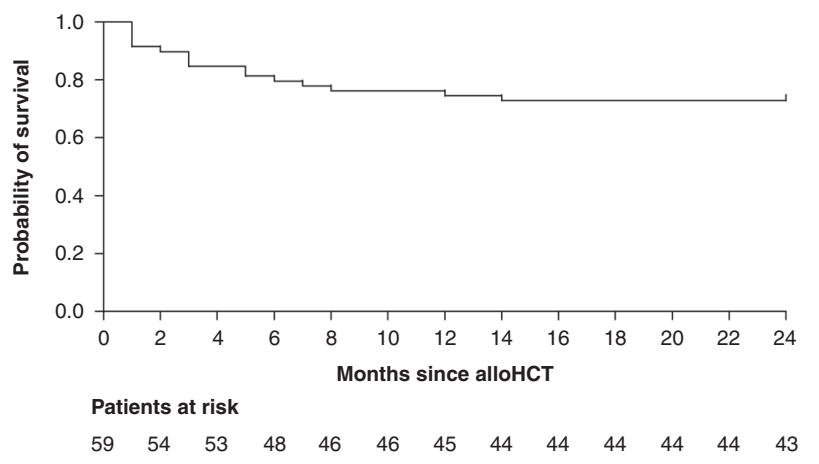

Fig. 1 Kaplan-Meier curve presenting overall survival of 59 analyzed patients. Additionally patients at risk are presented in a twomonth interval.

\section{Clinical outcomes}

During the observation period (24 months) 16 of 59 patients died. The median survival time of deceased patients was 3.3 months (0.6-14 months). Forty-three patients were still alive and thus censored after 2 years. Median OS $(50 \%$ of patients died) was not reached (Fig. 1). Univariable and multivariable analyses showed that VO2peak was not associated with either OS (Table 3) or NRM (Table 4). Same results are shown for three of four risk scores, age,
KPS, BMI, phase angle, and total energy consumption. However, the rDRI, FM, LBM, and BCM revealed associations with OS and NRM in univariable analyses (rDRI: high risk HR $0.184,95 \%$ CI $0.062-0.544, p=0.002$; very high risk HR $0.078,95 \%$ CI $0.015-0.395, p=0.002$, SHR 0.076, 95\% CI 0.009-0.641, $p=0.018$; FM: HR 0.974, 95\% CI $0.954-0.995, p=0.014$; SHR $0.974,95 \%$ CI $0.965-0.983, \quad p \leq 0.001 ; \quad$ LBM: HR $0.977,95 \%$ CI $0.956-0.997, p=0.026$; SHR 0.976, 95\% CI 0.967-0.985, $p \leq 0.001$; BCM: HR 0.975 , 95\% CI $0.955-0.996, p=$ 0.021 , SHR 0.975, 95\% CI 0.966-0.984, $p \leq 0.001$ ). Remission was only associated with OS (HR $0.315,95 \%$ CI 0.101-0.979, $p=0.049$ ). Multivariable models confirmed the significant association between rDRI and OS (very high risk HR 18.007, 95\% CI 3.878-83.602, $p \leq 0.001$ ) and NRM (high risk SHR 0.088, 95\% CI 0.027-0.281, $p \leq$ 0.001; very high risk SHR 0.018, 95\% CI 0.003-0.098, $p \leq$ 0.001). Patients in the very high risk group had a 18 times higher mortality risk than those of the low/ intermediate risk group. The male sex also increases overall mortality by a factor of six (HR 6.668, 95\% CI, 1.440-30.882, $p=0.015$ ).

\section{Physical conditioning}

Most of our patients (88\%) performed CPET till exhaustion quantified by RER $\geq 1.1$ or a heart rate $\geq 85 \%$ of the age predicted maximum. We detected no difference between 
Table 3 Cox regression analyses-association of pre-transplant variables with overall survival.

\begin{tabular}{|c|c|c|c|c|}
\hline & \multirow[t]{2}{*}{$\mathrm{HR}$} & \multicolumn{2}{|c|}{ CI $95 \%$} & \multirow[t]{2}{*}{$P$ value } \\
\hline & & Lower & Upper & \\
\hline \multicolumn{5}{|l|}{ Univariable analyses } \\
\hline VO2peak $[\mathrm{ml} / \mathrm{min} / \mathrm{kg}]$ & 0.937 & 0.628 & 1.400 & 0.752 \\
\hline Gender $^{\mathrm{a}}$ & 0.457 & 0.130 & 1.606 & 0.222 \\
\hline Age [years] & 1.030 & 0.991 & 1.072 & 0.137 \\
\hline Remission $^{\mathrm{b}}$ & 0.315 & 0.101 & 0.979 & $0.046^{*}$ \\
\hline Conditioning $^{\mathrm{c}}$ & 0.491 & 0.112 & 2.161 & 0.347 \\
\hline rPAM score [score] & 1.082 & 0.996 & 1.176 & 0.063 \\
\hline \multicolumn{5}{|l|}{ HCT-CI ${ }^{\mathrm{d}}$} \\
\hline Intermediate risk & 0.673 & 0.197 & 2.301 & 0.528 \\
\hline High risk & 0.762 & 0.242 & 2.400 & 0.642 \\
\hline \multicolumn{5}{|l|}{ EBMT score ${ }^{d}$} \\
\hline Intermediate risk & 0.898 & 0.174 & 4.630 & 0.897 \\
\hline High risk & 0.773 & 0.259 & 2.307 & 0.644 \\
\hline \multicolumn{5}{|l|}{$\mathrm{rDRI}^{\mathrm{e}}$} \\
\hline High risk & 0.184 & 0.062 & 0.544 & $0.002^{* *}$ \\
\hline Very high risk & 0.078 & 0.015 & 0.395 & $0.002^{* *}$ \\
\hline KPS $<90 \%$ & 1.452 & 0.468 & 4.507 & 0.519 \\
\hline$B M I\left[\mathrm{~kg} / \mathrm{m}^{2}\right]$ & 0.968 & 0.853 & 1.099 & 0.619 \\
\hline Fat Mass $[\%]^{10}$ & 0.974 & 0.954 & 0.995 & $0.014^{*}$ \\
\hline Lean Body Mass $[\%]^{10}$ & 0.977 & 0.956 & 0.997 & $0.026^{*}$ \\
\hline Body Cell Mass $[\%]^{10}$ & 0.975 & 0.954 & 0.996 & $0.020^{*}$ \\
\hline Phase angle standardized $\left[{ }^{\circ}\right]^{\mathrm{f}}$ & 0.980 & 0.610 & 1.575 & 0.935 \\
\hline $\begin{array}{l}\text { Total energy consumption during } \\
\text { exercises/week [kcal] }\end{array}$ & 0.993 & 0.982 & 1.005 & 0.238 \\
\hline Quality of life [\%] & 1.013 & 0.985 & 1.041 & 0.370 \\
\hline Physical functioning [\%] & 1.003 & 0.975 & 1.031 & 0.851 \\
\hline \multicolumn{5}{|l|}{ Multivariable analysis } \\
\hline VO2peak $[\mathrm{ml} / \mathrm{min} / \mathrm{kg}]^{5}$ & 1.090 & 0.670 & 1.773 & 0.729 \\
\hline Gender $^{\mathrm{a}}$ & 6.668 & 1.440 & 30.882 & $0.015^{*}$ \\
\hline BMI $\left[\mathrm{kg} / \mathrm{m}^{2}\right]$ & 0.873 & 0.727 & 1.050 & 0.149 \\
\hline Body cell mass $[\%]^{10}$ & 1.010 & 0.981 & 1.039 & 0.496 \\
\hline \multicolumn{5}{|l|}{$\mathrm{rDRI}^{\mathrm{e}}$} \\
\hline High risk & 0.526 & 0.095 & 2.908 & 0.461 \\
\hline Very high risk & 18.007 & 3.878 & 83.602 & $<0.001^{* *}$ \\
\hline Conditioning $^{\mathrm{c}}$ & 0.272 & 0.056 & 1.320 & 0.106 \\
\hline
\end{tabular}

$H R$ hazard ratio, $C I$ confidence interval, VO2peak peak oxygen consumption, $r P A M$ revised pretransplant assessment of mortality, HCT-CI Hematopoietic Cell Transplantation-specific Comorbidity Index, EBMT European Society for Blood and Marrow Transplantation, $r D R I$ revised Disease Risk Index, KPS Karnofsky Performance Scale, $B M I$ body mass index, ${ }^{5}$ one unit are $5 \mathrm{ml} / \mathrm{min} / \mathrm{kg},{ }^{10}$ one unit are $10 \%$

$* p \leq 0.05 ; * * p \leq 0.01$.

${ }^{\mathrm{a}}$ Reference group $=$ female.

${ }^{\mathrm{b}}$ Reference group $=\mathrm{CR} /$ chronic phase

${ }^{\mathrm{c}}$ Reference group $=$ reduced conditioning protocol.

${ }^{\mathrm{d}}$ Reference group $=$ low risk, values of intermediate and high risk group are in relation to the low risk group.

${ }^{\mathrm{e}}$ Reference group $=$ low-intermediate risk, values of high risk and very high risk group are in relation to the low-intermediate risk group.

${ }^{\mathrm{f}}$ Deviation from reference values.

$2 \mathrm{yrS}$ and $2 \mathrm{yrD}$ across experienced and measured exhaustion -in the median, patients reported the level of effort as "very hard" and attained $89 \%$ of the maximum predicted heart rate. The VO2peak and maximum reached watt were also comparable. In median all patients reached $120 \mathrm{~W}$ $(60-280 \mathrm{~W})$ and a VO2peak in relation to body weight of $20 \mathrm{ml} / \mathrm{min} / \mathrm{kg}(12.1-44.8 \mathrm{ml} / \mathrm{min} / \mathrm{kg})$. Differences in body composition were only noted in the amount of FM with 2 yrS showing a greater FM (median 27\% (9-42\%); $24 \%$ $(9-32 \%), p=0.026)$. Energy consumption per week and KPS were also comparable (energy consumption/week: $2 \mathrm{yrsS} 9126 \mathrm{kcal}$, range $2771-27881 \mathrm{kcal} ; 2 \mathrm{yrD} 8060 \mathrm{kcal}$, range $1965-19488 \mathrm{kcal}, p=0.343$; KPS: $2 \mathrm{yrS}$ and $2 \mathrm{rsD}$ $90 \%$, range $70-100 \%, p=0.375$ ). Detailed descriptions of physical conditioning are found in Table 5.

\section{Risk scores}

Risk assessments relying on the EBMT score and the rDRI revealed most patients $(61 \%$ and $54 \%)$ carried an intermediate risk. In turn, using the HCT-CI resulted in the same number of patients having a low, intermediate or high risk. There were no differences between $2 \mathrm{yrS}$ and $2 \mathrm{yrD}$ in their allocation to different risk groups (EBMT score $p=0.768$; HCT-CI $p=0.550$, rDRI $p=0.139)$ or the median rPAM score $(p=0.192)$.

\section{Discussion}

The aim of the present analysis was to examine the explanatory power of pre-alloHCT VO2peak to predict OS and NRM two years after transplantation and to compare this risk prediction to that of commonly-used risk scores. We hypothesized that assessing VO2peak would improve the present risk prediction methods and thus precise post-alloHCT prognosis. Contrary to our assumptions, no association of VO2peak to OS or NRM was found. Only the male gender and the rDRI were associated with OS and NRM two years after transplantation. In univariable analyses, body composition showed an association with OS and NRM, but this could not be confirmed in multivariable models.

Our results stand in contrast to Wood et al. [20] and Kelsey et al. [19], who showed that OS and NRM are partly predictable via the pre-alloHCT VO2peak. However, comparing their patient groups to ours reveals remarkable differences in the numbers of deceased patients. While our mortality rate in this study was only $27 \% 24$ months after transplantation (24 month), Wood et al. [20] reported $37 \%$ after 100 days ( 3 month) and Kelsey et al. [19] 52\% after 25 months. We speculate that our study population was in better health and more physically fit. Compared to Wood el al. [20] our patients suffered from fewer comorbidities (HCT-CI $\geq 3$ of $37 \%$ vs. $50 \%$ ) and demonstrated a higher physical performance level, i.e., greater values in maximum reached power during CPET ( $120 \mathrm{~W}$ vs. $47.5 \mathrm{~W})$. However, compared to healthy individuals' reference values, our 
Table 4 Fine \& Gray model analyses-association of pre-transplant variables with NRM.

\begin{tabular}{|c|c|c|c|c|}
\hline & \multirow[t]{2}{*}{ SHR } & \multicolumn{2}{|c|}{ CI $95 \%$} & \multirow[t]{2}{*}{$P$ value } \\
\hline & & Lower & Upper & \\
\hline \multicolumn{5}{|l|}{ Univariable analyses } \\
\hline VO2peak $[\mathrm{ml} / \mathrm{min} / \mathrm{kg}]^{5}$ & 0.998 & 0.894 & 1.114 & 0.975 \\
\hline Gender $^{\mathrm{a}}$ & 0.173 & 0.022 & 1.354 & 0.095 \\
\hline Age [years] & 1.023 & 0.975 & 1.074 & 0.353 \\
\hline Remission $^{\mathrm{b}}$ & 0.515 & 0.163 & 1.623 & 0.257 \\
\hline Conditioning $^{\mathrm{c}}$ & 3.548 & 0.504 & 24.959 & 0.203 \\
\hline rPAM score & 1.050 & 0.952 & 1.157 & 0.331 \\
\hline \multicolumn{5}{|l|}{$\mathrm{HCT}-\mathrm{CI}^{\mathrm{d}}$} \\
\hline Intermediate risk & 0.565 & 0.096 & 3.325 & 0.528 \\
\hline High risk & 1.997 & 0.546 & 7.311 & 0.296 \\
\hline \multicolumn{5}{|l|}{ EBMT score ${ }^{d}$} \\
\hline Intermediate risk & 0.546 & 0.114 & 2.603 & 0.865 \\
\hline High risk & 0.866 & 0.165 & 4.556 & 0.865 \\
\hline \multicolumn{5}{|l|}{$\mathrm{rDRI}^{\mathrm{e}}$} \\
\hline High risk & 0.320 & 0.092 & 1.110 & 0.073 \\
\hline Very high risk & 0.076 & 0.009 & 0.641 & $0.018^{*}$ \\
\hline KPS $<90 \%$ & 1.430 & 0.382 & 5.352 & 0.595 \\
\hline BMI $\left[\mathrm{kg} / \mathrm{m}^{2}\right]$ & 0.990 & 0.864 & 1.134 & 0.886 \\
\hline Fat Mass $[\%]^{10}$ & 0.974 & 0.965 & 0.983 & $<0.001^{* *}$ \\
\hline Lean Body Mass $[\%]^{10}$ & 0.976 & 0.967 & 0.985 & $<0.001^{* *}$ \\
\hline Body Cell Mass $[\%]^{10}$ & 0.975 & 0.966 & 0.984 & $<0.001^{* *}$ \\
\hline Phase angle standardized ${ }^{\mathrm{f}}$ & 1.110 & 0.630 & 1.956 & 0.718 \\
\hline $\begin{array}{l}\text { Total energy consumption during } \\
\text { exercises/week [kcal] }\end{array}$ & 0.995 & 0.984 & 1.005 & 0.331 \\
\hline Quality of life [\%] & 1.014 & 0.983 & 1.047 & 0.378 \\
\hline Physical functioning [\%] & 1.013 & 0.990 & 1.036 & 0.282 \\
\hline \multicolumn{5}{|l|}{ Multivariable analysis } \\
\hline VO2peak $[\mathrm{ml} / \mathrm{min} / \mathrm{kg}]^{5}$ & 1.070 & 0.949 & 1.207 & 0.271 \\
\hline Gender $^{\mathrm{a}}$ & 0.064 & 0.008 & 0.530 & $0.011^{*}$ \\
\hline BMI $\left[\mathrm{kg} / \mathrm{m}^{2}\right]$ & 0.945 & 0.788 & 1.133 & 0.541 \\
\hline Body cell mass $[\%]^{10}$ & 1.002 & 0.981 & 1.024 & 0.835 \\
\hline \multicolumn{5}{|l|}{$\mathrm{rDRI}^{\mathrm{d}}$} \\
\hline High risk & 0.088 & 0.027 & 0.281 & $<0.001^{* *}$ \\
\hline Very high risk & 0.018 & 0.003 & 0.098 & $<0.001^{* *}$ \\
\hline Conditioning $^{\mathrm{c}}$ & 6.555 & 0.814 & 52.777 & 0.077 \\
\hline
\end{tabular}

SHR sub-distribution hazard ratio, $C I$ confidence interval, VO2peak peak oxygen consumption, $r P A M$ revised Pretransplant Assessment of Mortality, HCT-CI Hematopoietic Cell Transplantation-specific Comorbidity Index, EBMT European Society for Blood and Marrow Transplantation, rDRI revised Disease Risk Index, KPS Karnofsky Performance Scale, $B M I$ body mass index, ${ }^{5}$ one unit are $5 \mathrm{ml} / \mathrm{min} / \mathrm{kg}$,

10 one unit are $10 \%$.

$* p \leq 0.05 ; * * p \leq 0.01$.

${ }^{\mathrm{a}}$ Reference group $=$ female.

${ }^{\mathrm{b}}$ Reference group $=\mathrm{CR} /$ chronic phase.

${ }^{\mathrm{c}}$ Reference group $=$ reduced conditioning protocol.

${ }^{\mathrm{d}}$ Reference group $=$ low risk, values of intermediate and high risk group are in relation to the low risk group.

${ }^{\mathrm{e}}$ Reference group $=$ low-intermediate risk, values of high risk and very high risk group are in relation to the low-intermediate risk group.

${ }^{\mathrm{f}}$ Deviation from reference values.

study population's VO2peak was below the 5\% percentile $[27,33]$ indicating that our patients were not especially fit. Furthermore, Kelsey et al. [19] reported even higher
VO2peak values and maximum workload during CPET. Nevertheless, CPET-protocol differences can lead to diverging results $[34,35]$, which can hamper the comparability of patients' physical capacity.

The association of rDRI with OS we found reflects the assumption that disease-specific determinants can provide best prognosis [36, 37]. Accordingly, the risk of NRM predicted by the rDRI is lower. In general, pre-transplant disease features are more likely to predict relapse mortality, while patient-specific characteristics possess greater validity for transplant-related mortality [4]. A high rDRI risk group means a high tumor burden and therefore patients' organism is already weakened before alloHCT. This increases the mortality risk [38] which is also indicated by the association of remission status and OS we found in univariable analysis. Shouval et al. [5] described only the random to moderate reliability of pre-transplant risk scores. This is in line with our findings regarding to the remaining scores. The cohorts used for the development of the scores significantly influence the explanatory power. This leads to a better prediction of the rPAM score and HCT-CI for patients undergoing myeloablative conditioning therapy in contrast to patients receiving reduced conditioning protocols [5]. Since $75 \%$ of our sample received reduced intensity conditioning, this could be a reason for the missing association between those risk scores and OS as well as NRM.

An explanation why male patients had a higher mortality risk cannot be given. So far it could only be shown that male patients are more affected by complications when they receive female donor cells [39]. But this donor-recipient constellation only existed in four cases.

In univariable analyses higher lean body mass, body cell mass, and fat mass were shown to have an association with reduced mortality risk 2 years after transplantation. Since this correlations could not be confirmed in multivariable analyses, other variables than body composition seem to be decisive for survival of our sample. Nevertheless, in other investigations the pre-transplant loss of body weight was associated with a higher NRM and shorter OS [40]. Furthermore, a low proportion of fat mass in conjunction with low muscle mass before intense medical treatment can raise the mortality risk for cancer patients [41, 42]. Thus, having the appropriate body composition and standard BMI are essential to resisting the treatment-induced loss of body mass, and to withstanding drug toxicity during alloHCT $[23,24]$. We assume that not adjusting BMI for gender and age and categorizing values for analysis could have led to the missing association [23]. In addition, infusions may have influenced the measurement of body composition via bioelectrical impedance analysis [43].

For a comprehensive explanation of our results, aspects of statistical analysis must also be taken into account. We provided a suitable sample size $(N=59)$ and observed 16 
Table 5 Physical conditioning.

\begin{tabular}{|c|c|c|c|c|}
\hline & All $N=59$ & $2 \mathrm{yrsS} N=43$ & $2 \mathrm{yrsD} N=16$ & $P$ value \\
\hline BMI $\left[\mathrm{kg} / \mathrm{m}^{2}\right]^{\mathrm{a}}$ & $25(19-33)$ & $25(19-33)$ & $25(19-31)$ & 0.653 \\
\hline Fat Mass [\% of body weight $]^{\mathrm{a}}$ & $26(9-42)$ & $27(9-42)$ & $24(9-32)$ & $0.026^{*}$ \\
\hline $\begin{array}{l}\text { Lean Body Mass [\% of body } \\
\text { weight] }]^{\mathrm{a}}\end{array}$ & $74(56-91)$ & $73(58-91)$ & $77(69-91)$ & 0.137 \\
\hline $\begin{array}{l}\text { Body Cell Mass [\% of body } \\
\text { weight] }{ }^{\text {a }}\end{array}$ & $35(27-48)$ & $35(27-48)$ & $38(30-48)$ & 0.598 \\
\hline Standardized phase angle $\left[{ }^{\circ}\right]^{\mathrm{a}, \mathrm{b}}$ & $-1.2(-4.7-1.4)$ & $-1.1(-4.7-1.4)$ & $-1.4(-2.7-0.7)$ & 0.894 \\
\hline \multicolumn{5}{|l|}{ Cardiopulmonary exercise test } \\
\hline $\mathrm{VO} 2$ peak $[\mathrm{ml} / \mathrm{min} / \mathrm{kg}]^{\mathrm{a}}$ & $20(12.1-44.8)$ & $20.8(12-34)$ & $18.9(12.7-44.8)$ & 0.515 \\
\hline $\operatorname{Pmax}[\mathrm{W}]^{\mathrm{a}}$ & $120(60-280)$ & $120(60-240)$ & $120(70-280)$ & 0.392 \\
\hline $\mathrm{RER}^{\mathrm{a}}$ & $1.1(1-1.3)$ & $1.1(1-1.3)$ & $1.1(1-1.3)$ & 0.913 \\
\hline $\mathrm{RPE}^{\mathrm{a}}$ & $17(13-20)$ & $17(14-20)$ & $17(13-20)$ & 0.603 \\
\hline $\begin{array}{l}\text { Heart rate } \% \text { of age predicted } \\
\text { maximum }^{\mathrm{a}}\end{array}$ & $89(65-109)$ & $89(70-104)$ & $90(65-109)$ & 0.785 \\
\hline $\begin{array}{l}\text { Total energy consumption } \\
\text { during exercises/week [kcal }]^{\mathrm{a}}\end{array}$ & $8425(1965-27,880)$ & $9126(2771-27,881)$ & $8060(1965-19,488)$ & 0.343 \\
\hline Quality of life [\%] & $58.3(16.7-91.7)$ & $58.3(16.7-91.7)$ & $66.7(25-91.7)$ & 0.343 \\
\hline Physical functioning [\%] & $80(20-100)$ & $80(20-100)$ & $80(53.3-100)$ & 0.983 \\
\hline
\end{tabular}

$2 y r s S$ two-year-survivor, 2yrs D two-year-decedents, BMI body mass index, VO2peak peak oxygen consumption, Pmax maximum power, RER respiratory exchange ratio, $R P E$ received perception of exertion.

$* p \leq 0.05$.

${ }^{a}$ Median (range).

${ }^{\mathrm{b}}$ Deviation from standardized values.

events (deaths). As the statistical power crucially depends on the number of events, only large prognostic-factor effects are detectable as revealing statistical significance. Thus, the low number of events is a potential weakness in our analysis. However, as comparable investigations $[19,20]$ did not report the quality of their statistical analysis, it is difficult to classify both their and our results.

The present survival analysis is limited by the fact that our data were analyzed retrospectively and retrieved from a randomized controlled trial that enforced stringent exclusion criteria. That randomized controlled trial was not designed to predict clinical outcomes. Furthermore, collecting data retrospectively can lead to failures to capture relevant data that might not have been recorded.

To best of our knowledge, we are the first to investigate the predictive power of physical conditioning and commonly-used risk scores in relation to survival time within one sample. We could only confirm that the rDRI and male gender predict survival after alloHCT. However, unlike physical performance, these variables cannot be positively influenced and do not reflect all individual preconditions of each patient. Considering the growing numbers of increasingly elderly and frail alloHCT recipients, our results underline the need for further research to identify an all-encompassing and meaningful examination method for predicting patients' pre-alloHCT risk.
Acknowledgements The randomized controlled trial in which context data of this analysis were collected was funded by the Comprehensive Cancer Center Freiburg. The article processing charge was funded by the University of Freiburg in the funding program Open Access Publishing.

Funding Open Access funding enabled and organized by Projekt DEAL.

\section{Compliance with ethical standards}

Conflict of interest The authors declare that they have no conflict of interest.

Publisher's note Springer Nature remains neutral with regard to jurisdictional claims in published maps and institutional affiliations.

Open Access This article is licensed under a Creative Commons Attribution 4.0 International License, which permits use, sharing, adaptation, distribution and reproduction in any medium or format, as long as you give appropriate credit to the original author(s) and the source, provide a link to the Creative Commons license, and indicate if changes were made. The images or other third party material in this article are included in the article's Creative Commons license, unless indicated otherwise in a credit line to the material. If material is not included in the article's Creative Commons license and your intended use is not permitted by statutory regulation or exceeds the permitted use, you will need to obtain permission directly from the copyright holder. To view a copy of this license, visit http://creativecommons. org/licenses/by/4.0/. 


\section{References}

1. for the European Society for Blood and Marrow Transplantation (EBMT), Passweg JR, Baldomero H, Bader P, Basak GW, Bonini $\mathrm{C}$, et al. Is the use of unrelated donor transplantation leveling off in Europe? The 2016 European Society for Blood and Marrow Transplant activity survey report. Bone Marrow Transplant. 2018;53:1139-48.

2. Horan JT, et al. Reducing the risk for transplantation-related mortality after allogeneic hematopoietic cell transplantation: how much progress has been made? J Clin Oncol. 2011;29:805-13.

3. for the Infectious Diseases Working Party EBMT, Styczyński J, Tridello G, Koster L, Iacobelli S, van Biezen A, et al. Death after hematopoietic stem cell transplantation: changes over calendar year time, infections and associated factors. Bone Marrow Transplant. 2020;55:126-36.

4. Potdar R, et al. Prognostic scoring systems in allogeneic hematopoietic stem cell transplantation: where do we stand? Biol Blood Marrow Transpl. 2017;23:1839-46.

5. Shouval R, et al. External validation and comparison of multiple prognostic scores in allogeneic hematopoietic stem cell transplantation. Blood Adv. 2019;3:1881-90.

6. Gratwohl A. The EBMT risk score. Bone Marrow Transpl. 2012;47:749-56.

7. Sorror ML. Hematopoietic cell transplantation (HCT)-specific comorbidity index: a new tool for risk assessment before allogeneic HCT. Blood. 2005;106:2912-9.

8. Armand P, et al. Validation and refinement of the Disease Risk Index for allogeneic stem cell transplantation. Blood. 2014;123:3664-71.

9. $\mathrm{Au} \mathrm{BKC}$, et al. Reevaluation of the pretransplant assessment of mortality score after allogeneic hematopoietic transplantation. Biol Blood Marrow Transpl. 2015;21:848-54.

10. Soubani AO, Pandya CM. The spectrum of noninfectious pulmonary complications following hematopoietic stem cell transplantation. Hematol Oncol Stem Cell Ther. 2010;3:143-57.

11. Sun C-L, et al. Prevalence and predictors of chronic health conditions after hematopoietic cell transplantation: a report from the Bone Marrow Transplant Survivor Study. Blood. 2010;116:3129-39.

12. Chow EJ, et al. Cardiovascular hospitalizations and mortality among recipients of hematopoietic stem cell transplantation. Ann Intern Med. 2011;155:21.

13. Kyle UG, et al. Longitudinal follow-up of body composition in hematopoietic stem cell transplant patients. Bone Marrow Transpl. 2005;35:1171-7.

14. Swain DP, American College of Sports Medicine, American College of Sports Medicine, editors. ACSM's resource manual for Guidelines for exercise testing and prescription. 7th ed. Philadelphia: Wolters Kluwer Health/Lippincott Williams \& Wilkins; 2014. 862 p.

15. García-Hermoso A, et al. Muscular strength as a predictor of allcause mortality in an apparently healthy population: a systematic review and meta-analysis of data from approximately 2 million men and women. Arch Phys Med Rehabil. 2018;99:2100-e5.

16. Kodama S. Cardiorespiratory fitness as a quantitative predictor of all-cause mortality and cardiovascular events in healthy men and women: a meta-analysis. JAMA. 2009;301:2024.

17. Jones LW, et al. Peak oxygen consumption and long-term all-cause mortality in nonsmall cell lung cancer. Cancer. 2010;116:4825-32.

18. Jones LW, et al. Cardiopulmonary function and age-related decline across the breast cancer survivorship continuum. J Clin Oncol. 2012;30:2530-7.

19. Kelsey CR, et al. Cardiopulmonary exercise testing prior to myeloablative allo-SCT: a feasibility study. Bone Marrow Transpl. 2014;49:1330-6.
20. Wood WA, et al. Cardiopulmonary fitness in patients undergoing hematopoietic SCT: a pilot study. Bone Marrow Transpl. 2013;48:1342-9.

21. Wasserman K, editor. Principles of exercise testing and interpretation: including pathophysiology and clinical applications. 5th ed. Philadelphia: Wolters Kluwer Health/Lippincott Williams \& Wilkins; 2012. 572 p.

22. Sutherland TJT, McLachlan CR, Sears MR, Poulton R, Hancox RJ. The relationship between body fat and respiratory function in young adults. Eur Respir J. 2016;48:734-47.

23. Urbain $P$, et al. Body mass index and bioelectrical impedance phase angle as potentially modifiable nutritional markers are independent risk factors for outcome in allogeneic hematopoietic cell transplantation. Ann Hematol. 2013;92:111-9.

24. Le Blanc K, Ringdén O, Remberger M. A low body mass index is correlated with poor survival after allogeneic stem cell transplantation. Haematologica. 2003;88:1044-52.

25. Pahl A, Wehrle A, Kneis S, Gollhofer A, Bertz H. Whole body vibration training during allogeneic hematopoietic cell transplantation-the effects on patients' physical capacity. Ann Hematol. 2020;99:635-48.

26. Limbach $\mathrm{M}$, et al. Influencing factors of cardiorespiratory fitness in allogeneic stem cell transplant candidates prior to transplantation. Support Care Cancer. 2021;29:359-67.

27. Rapp D, Scharhag J, Wagenpfeil S, Scholl J. Reference values for peak oxygen uptake: cross-sectional analysis of cycle ergometry-based cardiopulmonary exercise tests of 10,090 adult German volunteers from the Prevention First Registry. BMJ Open. 2018;8:e018697.

28. Luks AM, Glenny RW. Clinical exercise testing. In: Murray and Nadel's textbook of respiratory medicine [Internet]. Elsevier; 2016. p. 436-e6. https://linkinghub.elsevier.com/retrieve/pii/ B9781455733835000269. Accessed Jun 2020.

29. Borg G. Psychophysical bases of perceived exertion. Med Sci Sports Exerc. 1982;14:377-81.

30. Kyle UG, et al. Bioelectrical impedance analysis-part I: review of principles and methods. Clin Nutr. 2004;23:1226-43.

31. Bosy-Westphal A, et al. Phase angle from bioelectrical impedance analysis: population reference values by age, sex, and body mass index. J Parenter Enter Nutr. 2006;30:309-16.

32. Kyle UG, et al. Bioelectrical impedance analysis-part II: utilization in clinical practice. Clin Nutr. 2004;23:1430-53.

33. Kaminsky LA, Imboden MT, Arena R, Myers J. Reference standards for cardiorespiratory fitness measured with cardiopulmonary exercise testing using cycle ergometry: data from the Fitness Registry and the Importance of Exercise National Database (FRIEND) registry. Mayo Clin Proc. 2017;92(Feb):228-33.

34. Faria EW, Parker DL, Faria IE. The science of cycling: factors affecting performance-part 2. Sports Med. 2005;35:313-37.

35. Egaña M, Smith S, Green S. Revisiting the effect of posture on high-intensity constant-load cycling performance in men and women. Eur J Appl Physiol. 2007;99:495-501.

36. Shouval R, et al. Prediction of allogeneic hematopoietic stem-cell transplantation mortality 100 days after transplantation using a machine learning algorithm: a European Group for Blood and Marrow Transplantation Acute Leukemia Working Party Retrospective Data Mining Study. J Am Soc Clin Oncol. 2015;33:3144-51.

37. Armand $\mathrm{P}$, et al. A disease risk index for patients undergoing allogeneic stem cell transplantation. Blood. 2012;120:905-13.

38. De Witte T, et al. Haematopoietic stem cell transplantation for patients with myelo-dysplastic syndromes and secondary acute myeloid leukaemias: a report on behalf of the Chronic Leukaemia Working Party of the European Group for Blood and Marrow Transplantation (EBMT): stem Cell Transplantation for Myelodysplastic Syndromes. Br J Haematol. 2000;110:620-30. 
39. Nannya $Y$, et al. The negative impact of female donor/male recipient combination in allogeneic hematopoietic stem cell transplantation depends on disease risk. Transpl Int. 2011;24:469-76.

40. Radujkovic A, et al. Pre-transplant weight loss predicts inferior outcome after allogeneic stem cell transplantation in patients with myelodysplastic syndrome. Oncotarget. 2015;6:35095-106.

41. Charette N, et al. Prognostic value of adipose tissue and muscle mass in advanced colorectal cancer: a post hoc analysis of two non-randomized phase II trials. BMC Cancer. 2019;19:134.

42. Hopkins JJ, et al. The impact of muscle and adipose tissue on long-term survival in patients with stage I to III colorectal cancer. Dis colon rectum. 2019;62:549-60.

43. Savastano $S$, et al. Validity of bioelectrical impedance analysis to estimate body composition changes after bariatric surgery in premenopausal morbidly women. Obes Surg. 2010;20:332-9. 